\title{
Traditional Medicine and Diabetes Care in Myanmar
}

\author{
Tint Swe Latt ${ }^{1}$ \\ Than Than Aye ${ }^{2}$ \\ Ei Sandar U. \\ ${ }^{1}$ Myanmar Diabetes Association, Yangon, Myanmar \\ ${ }^{2}$ Myanmar Society of Endocrinology and Metabolism, Yangon, \\ Myanmar \\ ${ }^{3}$ Department of Medical Education and ICT, University of Public \\ Health, Yangon, Myanmar \\ ${ }^{4}$ Department of Diabetes and Endocrinology, University of Medicine 2, \\ Yangon, Myanmar
}

\author{
Yin Yin Win ${ }^{4}$
}

Khin Thawdar Wint ${ }^{1}$

Ei Thinzar Khin ${ }^{1}$

J Soc Health Diab 2019;7:16-21

\begin{abstract}
Address for correspondence Tint Swe Latt, President, Myanmar Diabetes Association, Yangon, Myanmar (email: proftsl@gmail.com).
\end{abstract}

\author{
Abstract \\ Keywords \\ - traditional medicine \\ - complementary \\ medicine \\ - diabetes care \\ - Myanmar
}

According to the National Survey of Diabetes and Its Risk Factors conducted in 2014, Myanmar has a high burden of diabetes, as the prevalence of diabetes in adult population was $10.5 \%$ and the estimated number of adults with diabetes was 2.5 million. Diabetes care in the country is still far from satisfaction, as more than $50 \%$ of people with diabetes were unaware of their diagnosis, and access to the comprehensive diabetes care is generally available in the big cities only. Moreover, most people have to pay the cost of health care out of their own pocket, as the health insurance system and social security system are currently in their rudimentary stage. Because of these gaps in diabetes care and health financing system, a significant number of people resort to the traditional medicine, which is familiar to a significant proportion of the public and also being relatively more accessible with affordable cost. This article assesses the current situation of traditional medicine in diabetes care in Myanmar and attempts to make some suggestions to remedy the drawbacks of traditional medicine. It discusses ways to adopt an integrated approach to strengthen the practice of traditional medicine, in general, in the health care system of the country regarding the diabetes care.

\section{Introduction}

Diabetes is a personal and social calamity and imposes unacceptably high burdens on individuals, their families, and national economies. Diabetes poses an immense global challenge by the steady rise in its prevalence. ${ }^{1}$ Despite innovative scientific discoveries to advance our understanding of the pathology of diabetes mellitus and to develop novel approaches to therapy, the burden of diabetes continues to escalate and treatment often remains substandard. ${ }^{2}$ It is estimated that the global number of people with diabetes is 415 million in 2015 and can increase up to 642 million in 2040. ${ }^{3}$ In Myanmar, the national prevalence of diabetes was $10.5 \%$ for the adult aged between 24 and 65 years, and it was estimated that the total number of people with diabetes was at least 2.5 million in 2013-2014. ${ }^{4}$

As a lifelong and progressive illness, diabetes requires continued care by a multifaceted health care team, starting from primary health care (PHC) with defined referral pathways to secondary and tertiary care for complications. ${ }^{5}$ The traditional medicines are familiar to the public and also relatively more accessible with relatively low cost, especially in PHC level. It is required to determine the impact of complementary and alternative medicine (CAM) therapies, especially that of widespread popular herbal remedies, on diabetes management instead of ignoring the sociomedical phenomena taking place in the societies. ${ }^{6}$

This article aims to review the role of traditional medicine (TM) in diabetes care in Myanmar with a view to improve both diabetes care and TM, eventually leading to a better outcome for patients with diabetes.

\section{Role of Traditional Medicine in Health Care}

TM is the sum total of the knowledge, skills, and practices based on the theories, beliefs, and experiences indigenous to different cultures, whether explicable or not, used in the received

March 27, 2019

accepted

April 6, 2019
DOI https://doi.org/

$10.1055 / \mathrm{s}-0039-1692507$

ISSN 2321-0656.
C2019 Novo Nordisk Education Foundation
License terms

(이 (1) $\Theta \circledast$ 
maintenance of health as well as in the prevention, diagnosis, and improvement or treatment of physical and mental illness. The terms "complementary medicine" or "alternative medicine" are used interchangeably with TM in some countries. They refer to a broad set of health care practices that are not part of that country's own tradition and are not integrated into the dominant health care system. ${ }^{7}$

TM is recognized as one of the resources of PHC services to increase availability and affordability and to contribute to improve health outcomes including those mentioned in the Millennium Development Goals. ${ }^{8}$

\section{Role of Traditional Medicine in the Health System of Myanmar}

TM has been existed in Myanmar since time immemorial. ${ }^{9}$ Throughout the course of history of Myanmar civilization, use of TM by the public has been well established. Although its usage declined during the colonial period, there had been resurgence in popularity among the public during World War period due to scarcity of western medication. Community acceptance and utilization of TM has been increasing to this day. ${ }^{10}$

Currently, TM plays an important role in the health system of Myanmar. The government accords high importance and provides considerable support to TM. ${ }^{11}$

The Department of Traditional Medicine (DTM) was established as a separate department under Ministry of Health in 1989, with the objectives of providing comprehensive TM services to the entire nation and developing evidence-based practice of TM. There are many TM hospitals and clinics providing services across the country. ${ }^{12}$

As for the development of human resources for TM, the Institute of Traditional Medicine was established in 1979 and was upgraded to the University of Traditional Medicine in 2001. ${ }^{9}$ There are more than 9,000 registered TM practitioners in the country, though only 1,612 have degrees from public institutions. ${ }^{13}$

In 1996, the government promulgated the Traditional Drug Law, and one of the objectives of this law is "To enable the public to consume genuine quality, safe and efficacious traditional drugs."

Both public and private sectors manufacture TMs. DTM takes responsibility for public sector manufacturing in two department-owned factories, in Yangon and Mandalay. The private TM industry is also developing and conducting mass production according to good manufacturing practice (GMP) standards. There are more than 2,500 registered-private TM drug producers and about 15 TM factories in Myanmar, but only two factories work with GMP standard. ${ }^{14}$

Myanmar Traditional Medicine Council Law was enacted in 2000. One of the objectives of this law is "To supervise traditional medicine practitioners for causing abidance by the rule of conduct and discipline." At present, there are more than 6,000 TM practitioners registered under the said law. ${ }^{14}$

Scientific research projects such as botanical, chemical, pharmaceutical, pharmacologic, and clinical investigations are being conducted on traditional herbal drugs. The Department of Medical Research plays a main role of conducting research on safety and efficacy of these medicines. Research papers are presented at the Myanmar Health Research Congress and annual Traditional Medicine Conference. ${ }^{14}$

\section{Concepts of Diabetes in the Myanmar Traditional Medicine}

TM in Myanmar has flourished over thousands of years and proudly becomes a distinct entity. This emerged as a delicate, useful, and important subject of community health in preventive, curative, and rehabilitative aspects. ${ }^{9}$

Basic discipline of TM had been identified as follows ${ }^{11}$ :

1. The Desana system It is based on natural occurrence such as hot and cold. Its concepts are largely dependent on Buddhist philosophy, with the therapeutics use of herbal and mineral compounds and diet.

2. The Bethitzza system This system is based on Ayurvedic concepts with extensive use of herbal and mineral compounds to establish balance among three dosas, namely Kapha, Vataand, and Pitta.

3. The Astrological system It is based on the calculations of zodiac of stars, planets, and the time of birth and age. These calculations are linked to prescribed dietary practices.

4. The Vezzadara system This system is largely dependent on meditation and practices of alchemy. ${ }^{9}$

According to Desana system of medicine, causal agents of diabetes mellitus are coldness (Sheeta), hyperactivity of internal Prithvi, and external Apoabhandhana aggravation, leading to formation of Sheeta Prithvi Abhandhana or Sangahita (A-1) type of disease. The early stage of diabetes mellitus is described as Sangahita.

Ancient science of Ayurveda discussed diabetes at length thousands of years ago. The word Madhumeha is the combination of two terms madhu and meha. It indicates outflow of sweet urine. Diabetes is also known as Maharoga (major disease) as almost all parts of the body and every cell of human physiology are affected.

Ayurveda divides diabetes mellitus into two categories:

1. Genetic (sahaja) occurring in young age from the very beginning of life that has some similarities with the juvenile diabetes or insulin-dependent diabetes.

2. Acquires (apathyaja) due to an unhealthy lifestyle that occurs in old age and obese people and has similarities with type 2 diabetes mellitus.

In Aryuveda, Madhumeha diabetes is caused by the following situations:

1. Ahara (diet): Cool, sweet, oily, and astringent food or Snidha and Guru properties possessing foods are taken excessively.

2. Vihara (exercise): Lack of exercise and sedentary lifestyle.

3. Utu (environmental factors): Living in cool place and cool season.

4. Pacakagni (digestive power) and Dhatuagni derangement. ${ }^{11}$ 


\section{Current Situation of Traditional Medicine Usage in Myanmar}

In spite of availability of biomedical drugs for managing different types of diabetes mellitus, many patients with diabetes still use other complementary or traditional herbs. ${ }^{15,16}$ Patients with diabetes and other common chronic medical conditions were more likely to use CAM. Adjusting for covariates, patients with diabetes with and without comorbidities were 1.8 and 2 times, respectively, more likely to use CAM than general population. ${ }^{17}$

Nearly all-Asian nations, including Myanmar, have a strong belief, which is a kind of obstinacy, in natural healing and curing. Many people have a greater tendency to believe in herbal medicine than in modern drugs, and the self-medication with TM for diabetes mellitus is usually the first choice among their health-seeking behaviors. ${ }^{18,19}$

According to the close group preliminary survey undertaken in diabetes clinics of Yangon General Hospital, it was found out that more than $70 \%$ of patients took TM of various kinds prior to attending the tertiary clinics. ${ }^{20} \mathrm{~A}$ community-based descriptive study on KAP of Myanmar People on Traditional Medicine was conducted in 2007. Most respondents (99.3\%) across all layers stated that they do trust in TM. The practice of self-medication was more pronounced among females, especially older age $(60+$ years), with lower income level. Among those respondents, $55.7 \%$ believed that TM would achieve cure and $43.1 \%$ thought it would improve their health problems. The main reasons for using TM included trust (58.9\%), traditional belief (31.7\%), advice from someone (13.4\%), affordable price (7.3\%), and disbelief in other medicines (3.4\%).21,22 Treatment-seeking behavior of patients with diabetes is influenced by their health literacy, motivation, socioeconomic status, and peer advice.

Unregulated advertisements on TM and widespread use of social media nowadays also facilitate the spread of unfounded claims, which can lead to misconceptions among the public and enhance the use of drugs without sound evidence of safety and efficacy. Because the health care expenditure is borne mainly by out-of-pocket manners, affordability is one of the most important determining factors in seeking medical advice.

\section{Scientific Studies on Traditional Medicine}

Although the government encourages the manufacturers of TMs to produce drugs in scientific ways, there remains some significant weakness both in research on TMs and in application of the principle of evidence-based medicine. ${ }^{22}$

There have been 47 studies on the efficacy of commonly used herbal medicines for diabetes during the period of the past 25 years (1992-2017). ${ }^{23-26}$ Most studies are just preclinical studies and mainly are animal experiments. ${ }^{27-32}$

There were only four community-based clinical trials (phase I studies). ${ }^{33-36}$ Although most studies found that tested drugs have efficacy in reducing blood glucose levels in acute setting, these studies were actually preclinical trials with small sample size and tested only within a short time. ${ }^{37-40}$ Phases II and III clinical studies on these herbal medicines were seldom conducted. There have been only a few studies on identification of active ingredients responsible for the glucose reduction..$^{41,42}$

Moreover, there have been few trials studying the benefits, adverse effects, and safety on long-term use. There were no post marketing studies on the tested drugs. Although the findings of the studies were usually presented in the Myanmar Health Research Congress, there is no other means of data dissemination and utilization. In fact, there has been a huge gap between research and data utilization in the real practice.

\section{Untoward Consequences of unregulated Traditional Medicine Practice and Drugs}

Although there has been paucity of the studies on the impact of TM practice and drugs in diabetes care in Myanmar, the following are the common observations and concerns by practicing endocrinologists regarding unregulated TM and its practices:

- False sense of security in patients who no longer seek proper diabetes care.

- Hyperglycemia from taking inefficacious medications leading to both acute and chronic complications.

- Hypoglycemia from adulterated medications that are actually mixture of some herbal medicines and western medicines (e.g., glibenclamide). ${ }^{43}$

- Drug-induced hepatitis, impaired renal function, and thrombocytopenia develop because of ingestion of unidentified ingredients in the herbal medicines.

- Lack of regular monitoring of glycemic status and risk factors for diabetes complications (e.g., cardiovascular diseases, nephropathy, and retinopathy) leading to disabilities and premature death unnecessarily.

Contrary to the expectation of positive contributions of TM practice and drugs to the health care system and well-being of patients with diabetes, these factors lead to negative impacts ultimately by causing socioeconomic burdens due to complications of diabetes.

\section{Challenges for the Practice of Traditional Medicine}

In spite of all the efforts of the government to improve the practice and safety of TM, there are still some challenges in improving the situation. Based on the global experiences, WHO has identified the challenges of the TM as follows:

- Development and enforcement of policy and regulations.

- Integration, in particular, identifying and evaluating strategies and criteria for integrating TM into national and primary health care (PHC).

- Safety and quality, notably assessment of products and services, qualification of practitioners, methodology, and criteria for evaluating efficacy.

- Ability to control and regulate traditional and complementary medicine (T\&CM) advertising and claims.

- Research and development; 
- Education and training of T\&CM practitioners;

- Information and communication, such as sharing information about policies, regulations, service and research data, or obtaining reliable objective information resources for consumers. ${ }^{\text {? }}$

Myanmar diabetes care has been facing all these challenges. However, safety and quality of herbals drugs are of primary concern. Control and regulation of the T\&CM advertising and claims should also be prioritized to prevent the public from being allured by misleading information. Existing research activities on TM should be scaled up to develop an evidence-based practice.

\section{How to Improve the Situation by cohesive and integrated Approach}

TM has been used widely until this 21st century in Myanmar with strong belief, which puts the role of TM not to be underestimated. Therefore, in line with the global trend and the guidelines from the WHO, role of TM in diabetes care in Myanmar should be strengthened.

Dr. Margaret Chan, Director-General of WHO, stated that "The two systems of traditional and western medicine need not clash. Within the context of primary health care, they can blend together in a beneficial harmony, using the best features of each system, and compensating for certain weakness in each."

WHO has set the goal for TM strategy 2014-2023 as

- Harnessing the potential contribution of T\&CM to health, wellness, and people-centered health care.

- Promoting safe and effective use of T\&CM through the regulation, evaluation, and integration of T\&CM products, practice, and practitioners into health system, as appropriate.

These goals will be reached by implementing three strategic objectives:

1. Building the knowledge base and formulating national policies.

2. Strengthening safety, quality, and effectiveness through regulation.

3. Promoting universal health coverage by integrating $\mathrm{T} \& \mathrm{CM}$ services and self-health care into national health systems. ${ }^{9}$

Therefore, it is clearly a huge responsibility for the Ministry of Health and government of Myanmar to build a cohesive and intergraded pathway for the practice of TM in order to provide safer and more effective options for the general population.

TM practitioners need to further upgrade their knowledge and skills in the light of current understanding and development of modern care for patients with diabetes. There should be opportunity for them to pursue continuous medical education and continuous professional development so that there will be more skilled, well-trained, licensed traditional medical practitioners instead of practitioners with skills handed down from generations to generations orally. Moreover, it is advisable for the University of Traditional
Medicine to improve and update the existing curriculum in aligns with the current knowledge of comprehensive diabetes care.

It is essential to do research of TM drugs on their quality, efficacy, and safety in both pre- and post-marketing phases. Research on TM drugs should not only aim at determining the efficacy of lowering blood glucose but also put an effort on identification of active ingredients from herbal drugs. These researches will contribute to approval for licensing in distribution and marketing. Hence, TM and Food and Drug Administration will be able to deliver proper information in commercial publicity.

All in all, it is vital to ensure for the public to have an access to safe and effective TMs and practice for comprehensive diabetes care. If TMs are safe and reliable, it would be really helpful to both patients and health care personnel as there would be one more option in choosing treatment plan for not only in diabetes but also in other diseases.

On the other hand, from the western medicine point of view, the effective pharmaceutical agents for glycemic control together with standard diabetes care in line with the evidence-based guidelines have already been developed by international professional associations. It is essential to improve and expand the existing western standard diabetes care system to all levels of health care across the country. It should be implemented at all levels of health care delivery. The standardized diabetes care with western medicine would be a better choice and should be encouraged to ensure effective glycemic control, thus preventing and delaying diabetes-related complications.

It is also essential to improve public awareness regarding benefits of western standard diabetes care and its effectiveness. Nevertheless, it is a big challenge to persuade all those who have a strong belief in TM to change their health-seeking behavior and discourage the practice of self-medication without diabetes care.

Meanwhile, TM may fill the gaps where availability of western medicines and care is limited, especially in some remote areas. To ensure that all TMs and practices are safe and efficacious for diabetes care to be in line with western treatment, it is imperative to develop a cohesive and integrated approach, inclusive of all concerned stakeholders, government, health care practitioners, patients, and public in the process. As addressed in the Beijing declaration, strengthening of communication between conventional and traditional medicine providers is needed for mutual understanding of their roles in modern diabetes care from both sides of parties.

\section{Conclusion}

In conclusion, both traditional and western medicines are useful in their own ways. It is unlikely to discourage the use of TM in treatment of diabetes in Myanmar as it has been well accepted and most people believe in it deeply. The most appropriate way is to make safe and reliable traditional medical practices based on research, evidence-based medicine, and development of appropriate national policy to implement more efficient and integrating TM into health 
care service regarding diabetes care. Giving health education on advantages and disadvantages of TM to the public is also imperative. On the other hand, standard diabetes care based on evidence-based western medicine should also be made known to the public as a better care. By integrating TM practice of good standard to the health care service, there should be positive outcomes for diabetes care in Myanmar.

\section{Conflict of Interest}

None declared.

\section{Acknowledgment}

The authors would like to heartily acknowledge Professor Than Maung, Rector (retired), University of Traditional Medicine, and Dr Thaung Hla, Director (Retired), Department of Medical Research, Yangon, for their assistance to find literature for Myanmar Traditional Medicine and their invaluable input to the article.

\section{References}

1 International Diabetes Federation. Global Diabetes Scorecard.http://www.idf.org/global-diabetes-scorecard/. Accessed December 25, 2016

2 Saaddine JB, Cadwell B, Gregg EW, et al. Improvements in diabetes processes of care and intermediate outcomes: United States, 1988-2002. Ann Intern Med 2006;144(7):465-474

3 International Diabetes Federation. IDF Diabetes Atlas. 7th ed. Brussels, Belgiu International Diabetes Federation; 2015. http:// www.diabetesatlas.org. Accessed February 18, 2016

4 Latt TS, Zaw KK, Aye TT, Ko K. Report on National Survey of Diabetes Mellitus and Risk Factors for Noncommunicable Diseases in Myanmar in 2014, Ministry of Health, Myanmar; 2014. Available at: https://www.who.int/ncds/surveillance/ steps/Myanmar_2014_STEPS_Report.pdf. Accessed 30 May 2019

5 Roglic G, Varghese C, Thamarangsi T. Diabetes in South-East Asia: burden, gaps, challenges and ways forward. WHO SouthEast Asia J Public Health 2016;5(1):1-4

6 Argáez-López N, Wacher NH, Kumate-Rodríguez J, et al; DIMSS Study Group. The use of complementary and alternative medicine therapies in type 2 diabetic patients in Mexico. Diabetes Care 2003;26(8):2470-2471

7 World Health Organization. WHO Traditional Medicine Strategy 2014-2023. World Health Organization; 2013

8 World Health Organization. Beijing Declaration. Beijing, China: WHO Congress on Traditional Medicine; November 8, 2008. Available at: http://www.who.int/medicines/areas/traditional/TRM_BeijingDeclarationEN.pdf. Accessed May 30, 2019.

9 Ministry of Health. Traditional Medicine in the Union of Myanmar. [Internet]. Searo.who.int. [Cited July 23, 2018]. Available at: https://apps.who.int/iris/bitstream/handle/ $10665 / 92455 / 9789241506090 \_$eng.pdf ? sequence $=1$. Accessed May 30, 2019.

10 Ministry of Health. Health in Myanmar 2014. Ministry of Health, Nay Pyi Taw;2014: 138-141. Available at: http://www. searo.who.int/entity/medicines/topics/,_medicine_in_union_ of_myanmar.pdf. Accessed May 30, 2019.

11 WHO Country Office for Myanmar Country Cooperative Strategy Myanmar, 2014-2018. WHO 2014. Available at: http://www.searo.who.int/myanmar/documents/HealthinMyanmar_2012_4_healthcaresystem. Accessed May 30, 2019.

12 Sein TT, Myint P, Tin N, Win H, Aye SS, Sein T. The Republic of the Union of Myanmar health system review (Health Systems in Transition, Vol. 4, No. 3, 2014). I. Asia Pacific Observatory on Health Systems and Policies. II. World Health Organization Regional Office for the Western Pacific; 2014
13 A Glance at Traditional Medicine in Myanmar | Myanmar Insider [Internet]. Myanmarinsider.com. [Cited July 23, 2018]. Available at: https://iris.wpro.who.int/bitstream/handle/ 10665.1/11354/9789290616665_eng.pdf. Accessed May 30, 2019.

14 Ministry of Health. Traditional Medicine in Myanmar. Ministry of Health 2012. Available at: http://www.searo.who.int/entity/medicines/topics/traditional_medicine_in_myanmar_ 2012.pdf. Accessed May 30, 2019.

15 Tchacondo T, Karou SD, Agban A, et al. Medicinal plants use in central Togo (Africa) with an emphasis on the timing. Pharmacognosy Res 2012;4(2):92-103

16 de-Graft Aikins A. Healer shopping in Africa: new evidence from rural-urban qualitative study of Ghanaian diabetes experiences. BMJ 2005;331(7519):737

17 Egede LE, Ye X, Zheng D, Silverstein MD. The prevalence and pattern of complementary and alternative medicine use in individuals with diabetes. Diabetes Care 2002;25(2):324-329

18 Zin T, Win NN, Oo TH, et al. Study on pattern of traditional health care utilization of the community in urban and rural areas of Taikkyi Township, Yangon Division. Research Congress, DMR, 1992

19 Latt TS, Tun S, Oo ES. Treatment seeking behavior of diabetes mellitus patients in Hmaw Bi Township, Yangon Division, Myanmar. The First International Public Health Conference in Myanmar, 2013

20 Aye T, Oo E, Aung M. Diabetes mellitus in Myanmar: socio-cultural challenges and strength. J Soc Health Diabetes 2014;2(1):9

21 Tin N. A Study of KAP on Myanmar People on Traditional Medicine. 55th Myanmar Medical Conference;2009:78-79

22 Zin T, Hla T, New N. Study of knowledge, perception and practice of patient utilizing traditional medical care at the traditional medicine centers in Yangon and Mandalay. Paper read at Myanmar Medical Research Congress, Scientific paper reading session. December 1991

23 Myint KY. The prevention and management of type 2 diabetes mellitus in Myanmar. Doctorate Thesis Submitted to Board for Degree of Doctor of Medicine, University of Medicine, October 2,2000

24 Swe S, Thi KM, Htway EE, et al. Acute toxicity and blood glucose lowering effect of aqueous extract of Bergenia ligulata leaf on laboratory based experimental animals. 41st Myanmar Health Research Congress; 2012:52

25 Aung NT, Chit K, Kyaing KO, Zaw MT, Aye TM. Clinical study of Gynuraprocumbers (Lour.) on type 2 diabetes patients of Myanmar. 41st Myanmar Health Research Congress; 2012:52-53

26 Myint MM, Than MA, Lwin KK, et al. Hypoglycemic effect of Phyllanthus niruri Linn. (Taung-ze-phyu) on rabbit model. Myanmar Health Research Congress; 2007:51-52

27 Kyaw AK, Thein TT, Phyu KP, , et al Pre-clinical safety and hypoglycaemic activity of ethanolic extract of Andrographis paniculata Nees. Myanmar Health Research Congress; 2008:37

28 Swe S, Nyi KS, Maw HH, et al. Hypoglycemic effect of Plantago major L. and Heliotropium indicum L. on albino rats. Myanmar Health Research Congress;2009:17-18

29 Aung S, Than MA, Lwin KK, Myint MM. Preliminary study of hypoglycaemic effect of Curcuma longa Linn. (Turmeric) rhizomes on rabbit model. Myanmar Health Research Congress; 2010:18-19

30 Oo ZH, Aung K. Hypoglycemic Effect of Syzygiumcumini (L.) Skeels. (Thabye) Bark by Using Adrenaline Induced Hyperglycemic Albino Rat Model. 19th Myanmar Military Medical Conference; 2012:16

31 Aung EPP, Lwin SH, Aye NN, Phyu KP. Hypoglycemic effect of Terminalia chebula Retz. (Phan-kha-thee) on diabetic albino rat Models. 45th Myanmar Health Research Congress, Yangon: DMR; 2017:62 
32 Aung HP, Kyaw M. Antihyperglycemic effect of Piper betle Linn. (Kun-Ywet) in alloxan-induced diabetic rats. 21st Myanmar Military Medical Conference; 2014:13-14

33 Zin T, Win NN, Myint W, et al. Clinical trial of antidiabetic traditional medicine formulation (TMF) No. 32. J Myanmar Health Sci Res 1994;1:11-17

34 Hla T, Win MM, Htay TT, Lwin YY, Thwe Y, Wint MM. Hypoglycemic potential of Momordica charantia Linn. on maturity onset diabetes mellitus patients. Myanmar Health Research Congress, Yangon, DMR (LM);1996;63

35 Wynn TT, Phyu KP, Oung MT, et al. Blood glucose lowering effect of Traditional Medicine Formular No. 28 (TMF28) on type 2 diabetes mellitus patients. Myanmar Health Research Congress. 2009; p21. 2009

36 Thwin TG, Kyaw MTD, Ohnmar M, et al. Effect of pomegranate juice consumption on oxidative stress and lipoprotein oxidation, aggregation and retention in diabetic patients. Myanmar Health Research Congress; 2010:41-42

37 Than A, Myint T, Myint MM, Myint W, Bwin M. Study of inhibitory effects of some traditional medicine formulations on adrenaline-induced hyperglycemia in animal models. Medical Research Congress; 1991:31

38 Thein TT, Maung M, Phyu KP, et al. Experimental study on acute anti-hyperglycemic activity of ethanolic extract of Andrographis paniculata Nees. on rats. Myanmar Health Research Congress; 2007:23
39 Htut MTD. A study on hypoglycemic activity of Coccinia indica (ivy gourd) (Kin-Pon Thee) on albino rats. 54th Myanmar Medical Conference; 2008:63

40 Swe S, Htway EE, Chan N, et al. Blood glucose lowering effect of Tecoma stans (L.) H.B.K. on albino rats. Myanmar Health Research Congress; 2010:19

41 Myint KTY, Myint SH, Zin T, Than MA, Myint MM, Khine MM, Myint MM, Myint SS. Antihyperglycemic activity and related chemical constitutes of Premna integrigolia Linn. (Taung-Tan-Gyi). Myanmar Health Sci Res J 2012;24(1):35-40

42 Hla EM, Myint ML, Than MA, Myint KTY, Myint MM. Anti-hyperglycemic effect of the leaves of Moringa oleifera Lam. and identification of quercetin compound. Myanmar Health Sci Res J (Golden Jubilee Publication) 2013;25(1):67-73

43 Chew HM. Woman hospitalized after consuming adulterated 'health product' touted as diabetes cure [Internet]. The Straits Times. 2018. [Cited July 23, 2018]. Available at: https://www. straitstimes.com/singapore/health/woman-hospitalisedafter-consuming-adulterated-health-product-touted-asdiabetes. Accessed May 30, 2019. 